\title{
Research on the effectiveness evaluation method of VLF Communication Jamming
}

\author{
Guangming Li \\ Navy Submarine Academy \\ Qingdao,China
}

\author{
Guangyou Cai \\ Navy Submarine Academy \\ Qingdao,China
}

\author{
Cuihua Liu \\ Navy Submarine Academy \\ Qingdao,China
}

\begin{abstract}
This paper introduces the communication jamming suppressing zone to evaluate the vlf communication jamming effectiveness, and proposes to make use of the jamming equation to set up the mathematical model of calculating the jamming suppressing zone.The calculation of jamming suppressing zone will provide the zone of effectively jamming intuitively, so the communication department can improve the understanding of the information in battlefield space. Then the communication department can get the cognition advantage of combat spatial and make the right decision timely.
\end{abstract}

Keywords-VLF;communication suppressing zone;jamming effectiveness

jamming;jamming

\section{INTRODUCTION}

The bit error rate of receive information can be treated as the indicator of communication jamming effectiveness assessment in the process of quantitative analysis of vlf communication jamming effectiveness, it reflects the impact of communication jamming on the quality of the communication system to transmit information, so it is a reasonable indicators of assessment. The reproduction of error rate in the two-dimensional coordinates is jamming suppressing zone. The calculation of the jamming suppressing zone can more intuitive give the geographical range of jamming on communication systems, so the communication department can improve the understanding of the information in battlefield space, and get the cognition advantage of combat spatial. Then the communication department can make the right decision timely.

\section{THE SUPPRESSION FACTOR AND JAMMING EQUATION}

The main object of researching is the jamming suppression factor in the process of quantitative analysis of the communications jamming effectiveness. The jamming suppression factor is defined as the ratio of communication receiver input power and signal power when the jamming is effective. To achieve effective interference, the bit error rate of communication receiver should reach a certain extent, affect the correct to receive, it is said that the communication is effectively suppressed. So the suppression factor is defined as the ratio of the interference power and signal power when the bit error rate reaches the limits of receiving correctly, it is denoted by $K_{a}$.

The jamming equation is the relationship of the position and energy of the communications transmitter, receiver and jammer when communication is effective interference.

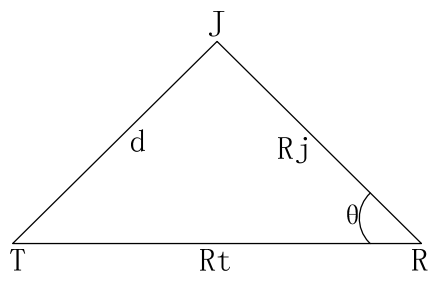

Figure 1. The spatial relationship of the transmitter, receiver and jammer

In figure $1, T, R$ and $J$ indicate the location of communications transmitter, receiver and jammer respectively, $R t$ is the communication distance, $R j$ is the distance between jammer and receiver, $d$ is the distance between jammer and transmitter, $\theta$ is the angle of the communication receiver to the transmitter and jammer. The ratio of jamming and signal power at the receiver should be more than the suppression factor when the jamming is effective, that is

$$
\frac{P_{r j}}{P_{r t}} \geq K_{a}
$$

\section{COMMUNICATION JAMMING SUPPRESSING ZONE}

In the VLF communication confrontation, if one side wants the jamming to be success, it must make the ratio of jamming and signal power enter the VLF receiver greater than the requirements to suppress system, that is to meet jamming equation.

After the vlf transmitter and jammer are determined, in the natural communication area which is defined as $Q_{N}$, the part in which the formula(1) is meted is called the communication jamming suppressing zone, which is defined as $Q_{y}$. If the region formed by the vlf receiver which can meet the formula(1) is defined as $Q_{y}^{\prime}$, so

$$
Q_{y}=Q_{y}^{\prime} \cap Q_{N}
$$

From this definition we can see that, when the locations of the vlf transmitter and jammer are determined, the point ( $A \in Q_{y}$ ) among communication jamming suppressing zone has the following properties:

No jamming, the receiver can communication smooth at the point A; When there is jamming, the VLF receiver at point A can not make smooth communication.

So $Q_{y}$ is the region that communication is suppressed, then it is called communication jamming 
effectively suppressing zone. It gives intuitively from the geometry communications jamming effectively suppressed region. The calculation of communication jamming effectively suppressing zone must calculate the boundary of the suppressing zone at first. At the boundary, the locations and power of the transmitter, receiver and jammer must meet the jamming equation:

$$
\frac{P_{j} G_{j} q_{r j}(\theta) \gamma_{j} \vartheta_{j}\left(R_{j}\right) B_{r j}}{P_{t} G_{t} q_{r t} \vartheta_{t}\left(R_{t}\right)}=K_{a}
$$

where:

$\Delta f_{r}$-receiver bandwidth;

$P_{j}$-jamming power;

$G_{j}$-jamming gain;

$R_{j}$-the distance between the jammer and receiver;

$q_{r j}(\theta)$-receive gain;

$r_{j}$-the polarization loss of jamming;

$P_{t}$-transmitter power;

$G_{t}$ - transmitter gain;

$q_{r t}$ - receiver power;

$\Delta f_{j}$-jamming bandwidth;

$R_{t}$ - the distance between the transmitter and receiver;

$K_{a}$-suppression system.

\section{The CALCUlATION OF JAMMING SUPPRESSING ZONE} defined as

The attenuation of vlf radio wave propagation can be

$$
\vartheta_{t}\left(R_{t}\right)=\frac{K}{R_{t}^{2}}
$$

Where $K$ is a constant, $R_{t}$ is the propagation distance between transmitter and receiver.

Therefore, the jamming equation can be turned into

$$
\frac{P_{j} G_{j} q_{r j}(\theta) R_{t}^{2} \gamma_{j} \Delta f_{r}}{P_{t} G_{t} q_{r t} R_{j}^{2} \Delta f_{j}}=K_{a}
$$

Let $B_{r j}=\frac{\Delta f_{r}}{\Delta f_{j}}$, according to the receiver antenna characteristics, shows that $q_{r j}(\theta)=q_{r t}$. Let $a^{2}=\frac{P_{j} G_{j} \gamma_{j} \Delta f_{r}}{P_{t} G_{t} \Delta f_{j} K_{a}}$,then formula(5) can be simplified to

$$
a^{2} R_{t}^{2}=R_{j}^{2}
$$

$\mathrm{T}$ is the location of transmitter, if $\mathrm{T}$ is the origin, the direction points to jammer $\mathrm{J}$ is defined as $\mathrm{X}$-axis, then we can set up the cartesian coordinate system showed in the figure 2 .

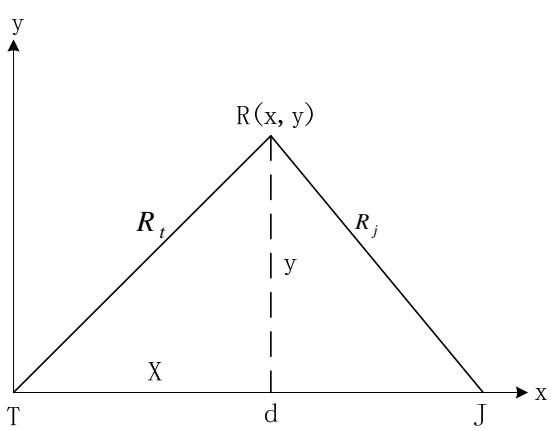

Figure 2. Cartesian coordinate system

Let the coordinate of the point on the boundary of communication jamming suppressing zone be (x,y),it follows that

$$
a^{2}\left(x^{2}+y^{2}\right)=(d-x)^{2}+y^{2}
$$

Where $d$ is the the distance between the jammer and transmitter.

If $a \neq 1$, thus

$$
\left(x-\frac{d}{1-a^{2}}\right)^{2}+y^{2}=\frac{a^{2}}{\left(1+a^{2}\right)^{2}} d^{2}
$$

This is a circle, its center is $\left(\frac{d}{1-a^{2}}, 0\right)$, its radius is $\frac{a}{\left|1-a^{2}\right|} \bullet d$.

\section{CONCLUSION}

1) If $a>1$, it shows that the jamming should be strong, the jamming suppressing zone is outside of the circle whose center is $O_{1}\left(\frac{d}{1-a^{2}}, 0\right)$, radius is $\frac{a}{a^{2}-1} d$. It is the smooth communication area within the circle. This shows that the communication should be smooth only in the circle around the transmitter when the jamming is stronger. But the center of the circle is not the point of $\mathrm{T}$, it is packed away from the side of the jammer.

2 ) If $a<1$, it shows that the jamming should be weak, the jamming suppressing zone is inside of the circle whose center is $\mathrm{O}_{2}\left(\frac{d}{1-a^{2}}, 0\right)$, radius is $\frac{a}{1-a^{2}} d$. It is the smooth communication area outside of the circle. The jamming suppressing zone becomes a closed area which is simply connected. 
3) If $a=1$, the boundary of the suppressing zone becomes $x=\frac{d}{2}$, it is the perpendicular bisector of the segment between the transmitter and jammer. The side close to the transmitter is the smooth communication area, the other side close to the jammer is the communication jamming suppressing zone.

\section{REFERENCES}

[1] Watt A D. VLF radio engineering[M]. Pregamon press, 1967.

[2] Jerry A, Long Wave Propagation Model[R],Naval Ocean Systems Center,1989.

[3] John E.Bickel, VLF/LF Propagation Measurements[C],AGARD,May 1993.

[4] Ferguson,J.A. and Snyder F.P.The Segnented Waveguide Program for Long Wavelength Propagation Calculations[R].Naval Ocean Systems Center San Dieho,California.1997.2.

[5] Pappert R.A. and Shockey L.R.Simplified VLF/LF Mode Coversion Program With Allowance for Elevated Arbitrarily Oriented Electric Dipole Antennas[R].1976. 